\title{
Probability in modal interpretations of quantum mechanics
}

\author{
Dennis Dieks \\ Institute for the History and Foundations of Science \\ Utrecht University, P.O.Box 80.000 \\ 3508 TA Utrecht, The Netherlands
}

\begin{abstract}
Modal interpretations have the ambition to construe quantum mechanics as an objective, man-independent description of physical reality. Their second leading idea is probabilism: quantum mechanics does not completely fix physical reality but yields probabilities. In working out these ideas an important motif is to stay close to the standard formalism of quantum mechanics and to refrain from introducing new structure by hand. In this paper we explain how this programme can be made concrete. In particular, we show that the Born probability rule, and sets of definite-valued observables to which the Born probabilities pertain, can be uniquely defined from the quantum state and Hilbert space structure. We discuss the status of probability in modal interpretations, and to this end we make a comparison with many-worlds alternatives. An overall point that we stress is that the modal ideas define a general framework and research programme rather than one definite and finished interpretation.
\end{abstract}

Key words: interpretation of quantum mechanics; modal interpretation; probability; many worlds

Email address: dieks@phys.uu.nl (Dennis Dieks). 


\section{Introduction: the modal point of view}

Modal interpretations of quantum mechanics are inspired by two main ideas. The first is to adopt a realist stance, in the specific sense of interpreting the theory's mathematical formalism in terms of properties and relations of physical systems, independent of whether or not human observers are around. The second motivating idea is that the relation between the formalism of quantum theory and physical reality is to be taken as probabilistic. That is, according to modal interpretations the quantum formalism does not tell us what actually is the case in the physical world, but rather provides us with a list of possibilities and their probabilities. The modal viewpoint is therefore that quantum theory is about what may be the case - in philosophical jargon, quantum theory is about modalities.

This viewpoint is at odds with the operational viewpoint that the quantum formalism should be interpreted as a recipe for predicting measurement outcomes. Modal interpretations strive for a description of the world in terms of objective, man-independent features, both on the macroscopic and (sub)microscopic level. These features may turn out to be monadic properties of physical systems (perhaps very exotic ones from a classical point of view), or perhaps rather a structural network of relations or perspectival properties; these are things to be decided during the detailed elaboration of the interpretation.

In accordance with this modal philosophy, measurement results are nothing but a subclass of the physical things existing in our world: positions of pointers attached to measuring devices, marks on computer tapes, etc. A measurement is a physical interaction between an object system and a measuring device, and should be treated like all other interactions. Modal interpretations therefore only need the quantum formalism with unitary evolution, i.e. the standard formalism without collapses. Indeed, as soon as the idea that measurements are something special is abandoned, the motivation for associating them with an own evolution mechanism, collapses, disappears; only the unitary (Schrödinger-like) evolution remains. Modal interpretations thus fall into the class of no-collapse interpretations of quantum mechanics.

So we assume that quantum mechanical states provide a description of physical systems. It is good to be explicit here about the distinction between the state as it is defined within the mathematical formalism (a vector in Hilbert space, or a density operator) and physical features of the represented systems - the latter are not mathematical entities. This distinction is important because it is not an a priori obvious matter what the exact relation between the mathematical state and physical reality is; it is not self-evident in what way the mathematical formalism represents. Discussions about the interpretation of quantum mechanics sometimes neglect this point by not clearly distinguishing 
between the formalism and what is represented by it (e.g., by accepting as self-evident that a +-sign in a superposition means joint existence).

Our task in modal interpretations is thus to endow the standard formalism, without collapses, with physical meaning. We need interpretational rules that tell us how the mathematical formalism relates to physical reality. Such rules do not constitute an addition to the formalism of quantum mechanics: they are not part of the mathematical formalism at all but establish a relation between this formalism and the world. Any interpretation of quantum mechanics will need to specify such a correspondence with reality. Mathematical theories cannot fix their own interpretation - as pieces of pure mathematics they do not contain information about their possible applications.

Quantum mechanics has a familiar history of enormously successful physical applications that make use of certain basic interpretational rules that have proved their mettle; there is no reason to doubt these. One of these basic interpretational rules is that physical quantities are represented by hermitian operators (observables). We will accept this standard correspondence (but will change some other rules).

A natural form our interpretational question now takes is: which physical quantities - represented by hermitian operators - can be assigned a definite value, when it is given that the physical system is represented by a specific mathematical state. Such definite values correspond to properties possessed by the system. It might turn out in later developments that it is more appropriate to focus on relations or perspectival properties instead of the monadic properties represented by definite values of physical quantities. But let us here focus on the standard modal line, which relies on the attribution of properties in the sense of definite values of physical quantities.

There may seem to be an easy answer to the question about the relation between states and properties. Standard quantum mechanics tells us that the state of a system is given by a density operator $W$, obtained by 'partial tracing' from the generally entangled state of the system and its environment. Now consider $W$ 's diagonal decomposition in terms of orthogonal projections:

$$
\begin{array}{r}
W=\sum_{i} p_{i}\left|\psi_{i}\right\rangle\left\langle\psi_{i}\right|, \\
\left\langle\psi_{i} \mid \psi_{j}\right\rangle=\delta_{i j} .
\end{array}
$$

This decomposition is unique if the coefficients $p_{i}$ are all unequal (the case of non-uniqueness will be discussed later on). There is a well-known way of interpreting such 'mixed states', namely via ignorance: according to it the physical system possesses one of the properties corresponding to the projectors $\left|\psi_{i}\right\rangle\left\langle\psi_{i}\right|$, but we don't know which one. In the special case of a pure state 
this reduces to the standardly accepted eigenstate-eigenvalue rule, according to which properties are only definite if the state is an eigenvector of the corresponding projection operator.

However, there are well-known objections to the general validity of this interpretation of $W$. The most important problem is that if it were true that the partial system possessed the property corresponding to $\left|\psi_{j}\right\rangle\left\langle\psi_{j}\right|$, then according to the eigenstate-eigenvalue link the system should be in the associated eigenstate $\left|\psi_{j}\right\rangle$. Analogously, the other partial system (the environment) must be in a pure state as well. But then a well-known theorem says that the total state must be the product state of these two pure states. This is in conflict with our initial assumptions: if the total state is $\left|\psi_{j}\right\rangle \otimes\left|\xi_{j}\right\rangle$, or a mixture of such states, there can be no entanglement whereas we assumed that in general the total state $i s$ entangled. The attribution of one of the properties $\left|\psi_{j}\right\rangle\left\langle\psi_{j}\right|$ therefore leads to contradictions.

To side-step this objection, modal interpretations propose to drop the rule that a system can only possess a well-defined value of a physical magnitude if it is represented by an eigenstate of the corresponding observable. In its stead comes a new interpretative principle according to which the mathematical state represents situations with definite physical properties even if this state is a superposition of eigenstates of the corresponding observables. The basic idea of interpreting the formalism in this vein has been put forward, with a number of variations, by several authors (van Fraassen, 1981, 1991; Kochen, 1985; Dieks, 1989a, b). Bas van Fraassen (1981), who seems to have been the first to think along these lines, coined the term 'modal interpretation'; but we still have to explain what the typically modal aspects are. Let us mention some more details in order to do so.

Consider the quantum mechanical treatment of a composite physical system, consisting of two parts. In this case, the total Hilbert space can be decomposed: $\mathcal{H}=\mathcal{H}_{1} \otimes \mathcal{H}_{2}$. According to a famous theorem (Schmidt, Schrödinger) there is a corresponding biorthogonal decomposition of every pure state in $\mathcal{H}$ :

$$
|\psi\rangle=\sum_{k} c_{k}\left|\psi_{k}\right\rangle \otimes\left|R_{k}\right\rangle
$$

with $\left|\psi_{k}\right\rangle$ in $\mathcal{H}_{1},\left|R_{k}\right\rangle$ in $\mathcal{H}_{2},\left\langle\psi_{i} \mid \psi_{j}\right\rangle=\delta_{i j}$ and $\left\langle R_{i} \mid R_{j}\right\rangle=\delta_{i j}$. This decomposition is unique if there is no degeneracy among the values of $\left|c_{k}\right|^{2}$.

One well-known version of the modal interpretation gives the following physical interpretation to this mathematical state. The system represented by vectors in $\mathcal{H}_{1}$ possesses exactly one of the physical properties associated with the set of projectors $\left\{\left|\psi_{k}\right\rangle\left\langle\psi_{k}\right|\right\}$, and definitely does not possess the others. That is, exactly one of the mentioned projectors is assigned the definite value 
1 , the others get the definite value 0 . The interpretation thus selects, on the basis of the form of the state $|\psi\rangle$, the projectors $\left|\psi_{k}\right\rangle\left\langle\psi_{k}\right|$ as definite-valued magnitudes. All physical magnitudes represented by maximal hermitian operators with spectral resolution given by $\Sigma a_{k}\left|\psi_{k}\right\rangle\left\langle\psi_{k}\right|$ are also definite-valued, since they are functions of the definite-valued projectors; their possible values are given by the functions in question applied to the values assumed by the projections.

In the case of degeneracy, that is $\left|c_{j}\right|^{2}=\left|c_{i}\right|^{2}$, for $i, j \in I_{l}$ (with $I_{l}$ a set of indices), the biorthogonal decomposition (11) still determines a unique set of projection operators, but these will generally be multi-dimensional. The onedimensional projectors have in this case to be replaced by projectors $P_{l}=$ $\sum_{i \in I_{l}}\left|\psi_{i}\right\rangle\left\langle\psi_{i}\right|$. The physical properties now correspond to this more general set of projectors. The general class of definite physical quantities contains in this case non-maximal hermitian operators in whose spectral resolution such multi-dimensional projectors occur.

We have just stipulated that only one of the values that can be assumed by the definite-valued observables is actually realized. This raises the question of what the probability is of the $l$-th alternative being actual. In accordance with the standard Born rule, this probability may be taken as $\left|c_{l}\right|^{2}$ (in the case of degeneracy this becomes $\sum_{i \in I_{l}}\left|c_{i}\right|^{2}$ ).

These details are mentioned here to clarify the spirit of the modal ideas. We will devote a more fundamental discussion to their justification, and to possible alternatives, later on. For example, how does the notion that only one possibility is realized compare to the many-worlds alternative, according to which all terms in the superposition correspond to actualities? And is it possible to derive and justify the Born probability rule, instead of just positing it?

Taking for granted the probabilistic character of the interpretation and the Born formula for the moment, we face the consequence that, in general, the physical situation could have been different from what it actually is, given the mathematical state. Here we have the 'modal' aspect of the interpretation: the mathematical state does not fix what is actual, but specifies what may be the case. It follows from the probabilistic nature of the relation between the state and the world that the same physical situation may be realized 'contingently' (if the associated probability is smaller than one; things could have been different in this case) or 'necessarily' (if the probability is one).

The just-explained interpretational rule ascribes definite physical properties to physical systems, even if the state is a superposition of eigenstates of the corresponding observables. This has the following consequence. According to the von Neumann measurement scheme, the situation after a measurement will 
typically be described by a superposition of the form (11), with $\left|\psi_{k}\right\rangle$ denoting states of the object system and $\left|R_{k}\right\rangle$ states of the measuring device ("pointer position states'). The modal interpretation of this state is that exactly one of the pointer positions is realized, with a probability given by the Born rule. This is the modal solution of the measurement problem: definite measurement outcomes are predicted even though there are no collapses of the wave function.

\section{Definite-valued observables}

Let us, after this review of the main ideas take a step back and look in a more systematic way at the interpretational possibilities that are left open by the motivating ideas behind the modal interpretation. This will make it clearer to what extent the just-discussed standard version can be justified, and will also enable us to say something about the modal interpretation's position among its competitors.

A core desideratum in devising the modal interpretation was the wish to deny a special status to measurements: measurements should be dealt with in the same way as ordinary physical interactions. Combined with the desire to keep the usual quantum formalism intact, this leads to a specific class of interpretations, namely no-collapse interpretations, in which there is only unitary evolution in Hilbert space. The task of all these interpretations is to link the unitarily evolving states in Hilbert space to physical features of the represented systems. The simplest programme for accomplishing this consists in the attempt to define a set of definite-valued observables from the mathematical state. These are observables that can be assigned a well-defined numerical value, and that thus fix physical quantities. Now, it is a notorious feature of the Hilbert space formalism that not all observables (i.e., hermitian operators) can be assigned definite values simultaneously (if we respect the functional relations between them); this follows from the Kochen-Specker theorem. The question therefore arises: What is the maximum set of observables that are definable from the quantum state and can jointly be given definite values without getting into contradictions? The usual quantum-Born - probabilities should become expressible as probability distributions over these definite values. Indeed, as already emphasized, measurement results are special cases of system properties in this approach, and the Born probabilities therefore come to pertain to the values of physical quantities - in the same way as probabilities in classical phase space.

In our search for definite-valued observables it is possible to include interpretations like the Bohm interpretation if we allow for the possibility that there is a preferred observable $R$ that is always definite, for all quantum states (in the Bohm theory position plays this role). The situation in which no privileged 
observable exists then becomes a special case. It should be noted, however, that there is a tension between this assumption of a preferred observable and the desideratum that the ordinary quantum formalism should be retained as much as possible: in the usual Hilbert space formalism there is no preferred observable. After discussing the case with a preferred observable we will therefore focus on the alternative, in which there is no such a priori fixed physical quantity, and in which the definite-valued observables are determined by the quantum state alone.

Consider an arbitrary pure quantum state represented by a ray $\psi$ in the Hilbert space $\mathcal{H}$. Let the Boolean algebra generated by the eigenspaces of the preferred observable $R$ be denoted by $\mathcal{B}(R)$. The usual quantum mechanical probabilities of the values of $R$, calculated via the Born rule applied to $\psi$, can be represented by an ordinary Kolmogorovian measure over the 2-valued homomorphisms (consistent assignments of truth values 0 and 1 ) on $\mathcal{B}(R)$. We now ask for the maximal lattice extension $\mathcal{D}(\psi, R)$ of $\mathcal{B}(R)$, formed by adding eigenspaces of other observables, such that we can represent in the same way the Born probabilities both for values of $R$ and for the values of these other observables.

Since we do not want to accept more mathematical structure not automatically present in Hilbert space than already introduced by the presence of $R$ as a preferred observable, we require these definite-valued observables to be definable solely in terms of $\psi$ and $R$. It follows that each element of $\mathcal{D}(\psi, R)$ should be invariant under all automorphisms of Hilbert space that preserve both the ray $\psi$ and the eigenspaces of $R$. This requirement (Dieks, 2005) is slightly stronger than the one made in (Bub and Clifton, 1996; Bub, 1997; Bub et al., 2000); we shall comment on the difference below. This invariance, expressing definability from $\psi$ and $R$, will do most of the work in determining our definite-valued observables.

Let us consider an $n$-dimensional Hilbert space $\mathcal{H}$, and an observable $R$ with $m \leq n$ distinct eigenspaces $r_{i}$ of $\mathcal{H}$. Let $\psi_{r_{i}}, i=1,2, \ldots, m$, denote the orthogonal projections of $\psi$ onto these eigenspaces $r_{i}$. Now, the set of automorphisms that leave $\psi$ and $R$ invariant includes all automorphisms that are equal to the unity operator when they operate on vectors orthogonal to $r_{i}$, and are rotations around $\psi_{r_{i}}$ or reflections with respect to the space orthogonal to $\psi_{r_{i}}$ inside $r_{i}$-since these transformations leave the eigenspaces of $R$ and the projections of $\psi$ the same, $R$ and $\psi$ themselves are invariant. Now consider a projection operator $P$ that is to correspond to a definite-valued property; so $P$ must be definable from $\psi$ and $R$. If the subspace of $\mathcal{H}$ on which $P$ projects is contained in one of the $r_{i}$, this subspace should therefore be invariant under the mentioned rotations and reflections with respect to $\psi_{r_{i}}$. This leaves four possibilities for the subspace in question: it can be the null-space, $\psi_{r_{i}}, \psi_{r_{i}}^{\perp} \wedge r_{i}$, or $r_{i}$. 
In the general case, the subspace on which $P$ projects will not be contained in one of the $r_{i}$ spaces, but will have non-zero projections on a number of them. The requirement that the subspace remains invariant under the abovementioned automorphisms now implies that its projections on the different spaces $r_{i}$ are each either null, $\psi_{r_{i}}, \psi_{r_{i}}^{\perp} \wedge r_{i}$, or $r_{i}$. All possible subspaces on which $P$ may project are therefore found by taking one of these latter spaces for each value of $i$, and constructing their span.

The lattice of subspaces that may correspond to definite propositions is therefore generated by all sublattices $\left\{0, \psi_{r_{i}}, \psi_{r_{i}}^{\perp} \wedge r_{i}, r_{i}\right\} 1$. In the case that $r_{i}$ is one-dimensional, $\psi_{r_{i}}$ is equal to $r_{i}$ and $\psi_{r_{i}}^{\perp} \wedge r_{i}$ equals 0 , so that the sublattice reduces to $\left\{0, r_{i}\right\}$.

It is clear from this construction that the resulting set of definite-valued projection operators is indeed a lattice: it is closed under the lattice operations of disjunction and conjunction (corresponding to taking the span or intersection of the associated eigenspaces). Moreover, the lattice is Boolean: all projection operators in it commute with each other. Therefore, no Kochen and Speckertype paradoxes can arise, and the quantum mechanical probabilities (including joint probabilities) can be represented by means of a classical measure on the lattice.

The above construction made use of the existence of a preferred observable, namely $R$. As stated above, it is important to see what happens if the state $|\psi\rangle$ and the Hilbert space structure are the only entities used to define the definite properties.

A possible way of implementing this is to take the projection on $|\psi\rangle$ itself for $R$. If we denote the subspace orthogonal to $\psi$ by $\psi^{\perp}$, we obtain the definite lattice consisting of the subspaces $\left\{0, \psi, \psi^{\perp}, \mathcal{H}\right\}$. The same result is obtained if we take the unity operator on $\mathcal{H}$ for $R$. This therefore leads to the 'orthodox' property assignment: only observables of which $|\psi\rangle$ is an eigenvector qualify as definite-valued (Bub, 1997). This traditional way of assigning properties returns us to the measurement problem, because after a measurement the combined system of measuring device and object system ends up in an entangled state that according to this assignment does not correspond to a definite pointer property. However, on second thought the situation is more complicated. The projection operator $|\psi\rangle\langle\psi|$ is an observable of the total system, and the just-mentioned property assignment pertains likewise to this total system. But we are really interested in the individual properties of device and object

1 The set of automorphisms that leave $\psi$ and $R$ invariant contains more elements than the ones considered in this derivation - so what has been proved is that the lattice of definite properties cannot be larger than the one constructed here. As the constructed lattice is clearly definable from $\psi$ and $R$ and satisfies the other requirements, it is the maximal lattice we were looking for. 
taken by themselves. Therefore, we need substitutes for $|\psi\rangle\langle\psi|$ that represent the states of these individual systems. In the context of standard quantum mechanics such operators are readily available, namely the density operators for the partial systems. Postponing for a moment possible doubts about the status of these operators in this new context, we are thus prompted to consider the definite lattices that result if the operators $W_{1} \otimes I$ and $I \otimes W_{2}$ are taken for $R$ (the total Hilbert space is the tensor product of Hilbert spaces belonging to the partial systems, $\left.\mathcal{H}=\mathcal{H}_{1} \otimes \mathcal{H}_{2}\right)$.

Denote the eigenspaces of $W_{1} \otimes I$ by $w_{l} \otimes \mathcal{H}_{2}, l=1,2, \ldots$ Now write $|\psi\rangle$ as a biorthogonal decomposition

$$
|\psi\rangle=\sum_{k, j} c_{k, j}\left|\alpha_{k, j}\right\rangle \otimes\left|\beta_{k, j}\right\rangle
$$

with $\left|\alpha_{k, j}\right\rangle$ in $\mathcal{H}_{1},\left|\beta_{k, j}\right\rangle$ in $\mathcal{H}_{2},\left\langle\alpha_{l, i} \mid \alpha_{m, j}\right\rangle=\delta_{l m} . \delta_{i j}=\left\langle\beta_{l, i} \mid \beta_{m, j}\right\rangle$. The second index, $j$, takes possible degeneracies into account: $\left|c_{k, j}\right|^{2}$ depends only on $k$, not on $j$. The projection of $|\psi\rangle$ on $w_{l} \otimes \mathcal{H}_{2}$ is given by $\left|\psi_{l}\right\rangle=\sum_{j} c_{l, j}\left|\alpha_{l, j}\right\rangle \otimes$ $\left|\beta_{l, j}\right\rangle$. As we have seen, it follows that the lattice of definite properties is generated by the sublattices $\left\{0, \psi_{l}, \psi_{l}^{\perp} \wedge\left(w_{l} \otimes \mathcal{H}_{2}\right), w_{l} \otimes \mathcal{H}_{2}\right\}$. We can restrict this lattice to a lattice of definite properties of the first system alone (represented in $\mathcal{H}_{1}$ ) by looking for those definite projections in the lattice that possess the form $P \otimes I$. The projection operators $P$ can in this case be taken to represent properties of system 1 by itself. Inspection of the lattice shows that all projections of the sought form are generated by the projectors $P_{w_{l}} \otimes I$. The restriction of the lattice of definite properties of the combined system to a lattice of definite properties of system 1 is therefore the Boolean lattice generated by the projections $P_{w_{l}}$. These are exactly the properties assigned by modal interpretations of the type discussed in the Introduction (Dieks, 1989a.,b; Vermaas and Dieks, 1995). In measurement situations these definite properties should correspond to pointer positions, 2

The analysis just given is similar to the one proposed by Bub and Clifton (Bub and Clifton, 1996; Bub, 1997; Bub et al., 2000). The difference is that these authors required the set of definite properties as a whole to be definable from $|\psi\rangle$ and $R$, whereas here we have imposed the stronger demand that the individual definite properties be so definable. Given the idea that $|\psi\rangle$ should fix as many elements of the interpretation as possible, our stronger requirement seems the more natural one; moreover, it makes the analysis considerably sim-

$\overline{2}$ It is an important question whether this requirement of empirical adequacy is in fact fulfilled. In situations with a limited number of degrees of freedom this has been shown to be the case (Bacciagaluppi and Hemmo, 1996); but there are grounds for doubt in cases in which the number of degrees of freedom is infinite or very large (Bacciagaluppi, 2000); see (Bene and Dieks, 2002) for a possible response). 
pler. As was to be expected, the lattice of definite properties that we found above on the basis of our stronger requirement is included in the lattice determined by Bub and Clifton. The latter possesses more 'fine structure': The Bub-Clifton lattice contains projection operators that cannot be defined individually but still belong to the set of projectors defined as a whole. However, these differences are not very significant. In the case of the measurement-like situation we have just discussed, the only difference is that in the Bub-Clifton approach all individual one-dimensional projections within the null-space of $W_{1}$ are definite, whereas in our approach it is only the total projector on this null-space that is definite-valued.

In this derivation we took $W_{1}$ for the state of system 1 . This is standard practice in quantum mechanics; however, the usual justification relies on the probabilistic interpretation of the theory and the Born rule. It would be preferable not to presuppose anything about this at the present stage. Indeed, in the next section we will make an attempt to derive the Born rule. It is therefore desirable to have a derivation of the definite-valued observables of the partial systems that does not presuppose that the density operators $W_{i}$ characterize the individual systems.

In order to achieve this we again make use of the biorthogonal way of writing $|\psi\rangle$, Eq. (2). As before, our aim is to determine maximal sets of properties of system 1 that can be defined from this state. We will use that $\mathcal{H}$ is the tensor product of the Hilbert spaces of the individual systems 1 and $2, \mathcal{H}=$ $\mathcal{H}_{1} \otimes \mathcal{H}_{2}$ : we want the definite properties of system 1 to be invariant under automorphisms that leave $|\psi\rangle$ the same and that respect this factorization of the total Hilbert space. These automorphisms have the form $U_{1} \otimes U_{2}$, with $U_{1}$ and $U_{2}$ defined on $\mathcal{H}_{1}$ and $\mathcal{H}_{2}$, respectively (Dieks, 1995, 2005). So we ask which automorphisms $U_{1} \otimes U_{2}$ leave $|\psi\rangle$ invariant, and which projectors in $\mathcal{H}_{1}$ remain the same under their operation (in other words, under the operation of the associated $U_{1}$ ).

To investigate this we must have a closer look at the invariance properties of (2). This biorthogonal decomposition is unique up to certain unitary transformations within the subspaces spanned by the vectors $\left\{\left|\alpha_{k, j}\right\rangle\right\}_{j}$ (and $\left\{\left|\beta_{k, j}\right\rangle\right\}_{j}$ ), with fixed values of $k$ (these are the 'degeneracy subspaces', labelled by values of $k$ ). This can be seen in the following way. The component of $|\psi\rangle$ within such a degeneracy subspace can (after normalization) be written as

$$
|\omega\rangle=\sum_{j} N^{-1 / 2} \exp i \phi_{j}\left|\alpha_{j}\right\rangle \otimes\left|\beta_{j}\right\rangle
$$

with $N$ the dimension of the subspace in question. Now take an arbitrary unitary operator $U_{I}$ in the subspace of $\mathcal{H}_{1}$ spanned by the vectors $\left|\alpha_{j}\right\rangle$. Define an operator $U_{I I}$ in the subspace of $\mathcal{H}_{2}$ spanned by the vectors $\left|\beta_{j}\right\rangle$, through 
its matrix elements, as follows:

$$
\left\langle\beta_{k}\left|U_{I I}\right| \beta_{l}\right\rangle=\overline{\left\langle\alpha_{k}\left|U_{I}\right| \alpha_{l}\right\rangle} \cdot \exp i\left(\phi_{k}-\phi_{l}\right)
$$

with the bar denoting complex conjugation. It follows from this definition that $U_{I I}$ is unitary (given the unitarity of $U_{I}$ ). We can now construct a product unitary operator in the tensor product of the two subspaces: $U=U_{I} \otimes U_{I I}$. This operator leaves $|\omega\rangle$ invariant:

$$
\begin{array}{r}
\langle\omega|U| \omega\rangle=N^{-1} \sum_{i, j} \exp i\left(\phi_{j}-\phi_{i}\right)\left\langle\alpha_{i}\left|U_{I}\right| \alpha_{j}\right\rangle\left\langle\beta_{i}\left|U_{I I}\right| \beta_{j}\right\rangle= \\
=N^{-1} \sum_{i, j}\left|\left\langle\alpha_{i}\left|U_{I}\right| \alpha_{j}\right\rangle\right|^{2}=1 .
\end{array}
$$

In other words, we can operate with an arbitrary unitary operator $U_{I}$ in one of the degeneracy subspaces of $\mathcal{H}_{1}$, and undo its effect on $|\psi\rangle$ by operating with a suitably chosen unitary operator $U_{I I}$, as defined in Eq. (4), in the corresponding degeneracy space of $\mathcal{H}_{2}$. It is of course also true that operating in a similar way with an arbitrary unitary in a degeneracy subspace of $\mathcal{H}_{2}$ can be undone by a corresponding unitary operation in $\mathcal{H}_{1}$.

In the case of a one-dimensional subspace (i.e., corresponding to a non-degenerated term in the superposition), $U_{I}$ can only be a multiplication by a phase factor, $\exp i \phi$. In this case the compensating $U_{I I}$ takes the form of multiplication by the inverse factor, $\exp -i \phi$.

Any spaces contained within the degeneracy subspaces selected by the biorthogonal decomposition are clearly not invariant under all unitary product operations in $\mathcal{H}=\mathcal{H}_{1} \otimes \mathcal{H}_{2}$ that preserve $|\psi\rangle$; only the degeneracy subspaces themselves are invariant. These spaces are exactly the eigenspaces of the reduced density operator $W_{1}$.

So we arrive in a quick and simple way at the same conclusion as before: the lattice of those properties of system 1 that can be defined on the basis of $|\psi\rangle$ alone, is generated by the projection operators $P_{w_{k}}$. Since this lattice is Boolean, definite values can be jointly assigned to all its elements without contradictions, and measures on the lattice can be represented in a classical Kolmogorovian probability space.

We have thus found a uniqueness result for the set of definite-valued observables. But any uniqueness result stands or falls with its premises. In our derivation we assumed that the definite-valued observables should be definable from $|\psi\rangle$ and the splitting of the total Hilbert space into two factor spaces, representing the system and its environment, respectively. If it is assumed that more or other ingredients play a role in determining the properties of a system, 
different definite observables will result. It has been suggested, for example, that we should not just look at the system and its environment, but rather at a three-fold splitting of the total Hilbert space into factors corresponding to the system, a measuring device (or, more generally, a system that is able to make records) and the remaining environment, respectively (Zurek, 2005; Schlosshauer, 2004). If the total state can be written as

$$
\left|\Psi_{s A \varepsilon}\right\rangle=\sum_{k} a_{k}\left|s_{k}\right\rangle\left|A_{k}\right\rangle\left|\varepsilon_{k}\right\rangle
$$

with $s, A$ and $\varepsilon$ referring to system, device and environment, respectively, with orthogonal pointer states $\left\{\left|A_{k}\right\rangle\right\}$, these orthogonal pointer states will be the only ones that make the three-fold factorization of (6) possible. In this way a set of preferred pointer states of the device can be defined. A problematic feature of this proposal is that it does not lead to a general assignment of properties to arbitrary systems; and that states of the form (6) are very special. Such states will only result from specific interactions (Zurek, 2005, sect. 4.2). In the context of decoherence studies it has indeed often been suggested that it is the form of the interaction Hamiltonian between (macroscopic) systems and their environments that does the selecting of preferred states: that pointer states are 'memory states' that behave in a robust and approximately classical way. The selected pointer observable commutes with the interaction Hamiltonian (perhaps in an approximate way), so that the environment effectively performs a non-demolition measurement of the pointer observable - see also Zurek (1993) 3 These proposals deserve further study. Questions to be asked are, e.g., about the presence of physical properties in cases without suitably interacting environments and recording devices. Can sense be made of the suggestion that the concept of physical properties becomes only applicable in special circumstances? Another issue is the status of the approximations that are usually involved in decoherence calculations (compare section 5 below). Anyway, it has to be admitted that decoherence proposals have led to plausible candidates for preferred states in many model calculations.

It should therefore be stressed that the modal ideas constitute a research programme rather than a completely fixed interpretation. The central features remain that the quantum formalism describes the world in man-independent terms, in particular without according a special role to measurements undertaken by humans; and that the relation between formalism and physical reality is probabilistic. This leaves room for differences in detailed elaborations.

3 Zurek (2005, sect. IIB) states, however, that all 'measurable properties' of a system can depend only on its own state, obtained by partial tracing from the entangled state of the system and its environment. This seems to lead us back to the standard modal property attribution. 


\section{The Born measure}

The modal interpretation is probabilistic and must therefore define a probability measure on the lattice of definite-valued observables. This raises the question: Is it possible to derive a preferred measure on the lattice of definitevalued observables, along the same lines as in the derivation of the definitevalued observables? More specifically: if we impose the requirement that the measure is to depend only on the state in Hilbert space, the tensor product structure of Hilbert space and the preferred observables induced by the state, has this enough bite to single out a definite form of the measure? An affirmative answer would fit in nicely with the modal philosophy according to which the standard quantum mechanical formalism is descriptively complete; that no elements need to be added by hand.

As we will argue, the answer is 'yes': the Born measure is the only one that is definable from just the relation between $|\psi\rangle$ and its associated definite-valued observables.

Denote the measure to be assigned to the definite-valued projector $P$, if the state is $|\psi\rangle$, by $\mu(|\psi\rangle, P)$. Write $|\psi\rangle$ in its biorthogonal form again:

$$
|\psi\rangle=\sum_{k} c_{k}\left|\alpha_{k}\right\rangle \otimes\left|\beta_{k}\right\rangle
$$

where we now have taken the non-degenerate case for simplicity. First note that we can take the coefficients $c_{k}$ to be real numbers: all phase factors can be absorbed into the vectors $\left|\alpha_{k}\right\rangle$ or $\left|\beta_{k}\right\rangle$, without any effect on the observables that are value-definite (the projection operators are invariant under this operation). So if $\mu$ is going to depend on the coefficients $c_{k}$, only their absolute values or, what amounts to the same thing, only $\left|c_{k}\right|^{2}$ can enter the expression.

An alternative road to this conclusion is to use the transformations $U_{I} \otimes U_{I I}$ under which $|\psi\rangle$ is invariant. As we have seen in the previous section, both $U_{I}$ and $U_{I I}$ are pure phase transformations in this non-degenerate case. One could now reason as follows, like Zurek (2005): any physical features pertaining to system $I$ alone should be invariant under the operation of any $U_{I}$ on $|\psi\rangle$, for the following reason. The effect of $U_{I}$ can be undone by $U_{I I}\left(U_{I}\right.$ is what Zurek calls an 'envariance' operation); and $U_{I I}$ should not be expected to affect the physical properties of $I$. Consequently, any effects $U_{I}$ may have on the mathematical state of $I$ should not be relevant to the physical features of $I$. In particular, the phases of the coefficients $\left\{c_{k}\right\}$ must be irrelevant, so that only their absolute values can count.

To conclude that $\mu$ indeed only depends on $\left\{c_{k}\right\}$, we need an additional argument, however. As pointed out by Caves (2005), it is not 'envariance' that 
is doing the work here: rather, the assumption (also made by Zurek) that the probabilities and physical properties pertaining to system $I$ do not depend on the vectors $\left\{\left|\beta_{k}\right\rangle\right\}$ in the biorthogonal decomposition is central-and once we make this assumption, the notion of envariance is no longer needed. This assumption may be seen as a no-signalling condition: its violation would make it possible to change physical features of $I$ by intervening in the state of $I I$, which would make it possible to signal. It can also be regarded as a non-contextuality condition: it should not make a difference for the characteristics of $I$ what unitary operations are taking place in its environment $I I$. This entails invariance of the probabilities under arbitrary $U_{I I}$. In particular, $\mu$ can only depend on system $I$ 's definite-valued projectors $P$ and on $\left\{c_{k}\right\}$, and since absorbing all phase factors into $\left\{\left|\beta_{k}\right\rangle\right\}$ does not change the probabilities only the absolute values of $\left\{c_{k}\right\}$ can be relevant-for further discussions of Zurek's line of argument see (Barnum, 2003; Caves, 2005; Mohrhoff, 2004; Schlosshauer and Fine, 2005). In brief, the non-contextuality condition entails that all probabilities must be invariant under application of arbitrary unitaries $U_{I} \otimes U_{I I}$. So we find that the bases $\left\{\left|\alpha_{k}\right\rangle\right\}$ and $\left\{\left|\beta_{k}\right\rangle\right\}$ are irrelevant for the probabilities, and only the values $\left\{\left|c_{k}\right|\right\}$ can play a role.

However, this irrelevance of $\left\{\left|\alpha_{k}\right\rangle\right\}$ and $\left\{\left|\beta_{k}\right\rangle\right\}$ for the expression of $\mu$ can be justified in a more direct way, without invoking principles about causality and contextuality, by a definability argument like the one in the previous section. We want $\mu$ to be definable exclusively from $|\psi\rangle$ and the product Hilbert space structure. We can therefore immediately impose the requirement that unitary transformations of the form $U_{I} \otimes U_{I I}$ should not change the values taken by the measure; that these values remain the same, but now apply to transformed projectors (like $U_{I}^{-1} P U_{I}$ ). The reason is that these unitary transformations only change the orientation of $|\psi\rangle$ in Hilbert space, but do not change anything in the relation between $|\psi\rangle$ and the definite-valued observables determined by it; all changes are equivalent to those induced by a basis transformation in the Hilbert space, and can be undone by performing an inverse basis transformation. But we want $\mu$ to be determined solely by the the state and its associated definite-valued projectors - the choice of a basis in Hilbert space in terms of which the state is written down should be immaterial. In other words, the same collection of $\mu$ values must be associated with the entire class of states that follow from $|\psi\rangle$ by application of arbitrary unitary operations $U_{I} \otimes U_{I I}$. Since the only feature that is common to all these states are the values of $\left|c_{i}\right|, \mu$ must be a function of these values only. As pointed out above, we can therefore consider $\mu$ to be a function of $\left\{\left|c_{i}\right|^{2}\right\}$.

Now compare the situation described by $|\psi\rangle$ with the one in which we discard, forget, or are unable to observe the differences between the different $\left|\beta_{k}\right\rangle$ for $k \geq 2$. The total probability of not having $\left|\beta_{1}\right\rangle$ should now be the sum of the probabilities of $\left|\beta_{k}\right\rangle$ for $k \geq 2$, since distinct alternatives have been grouped together. The vector that would correspond exactly to this new situation re- 
sults from $|\psi\rangle$ by erasing the differences between $\left|\beta_{k}\right\rangle$ for $k \geq 2$, and replacing all these vectors by $\left|\beta_{2}\right\rangle$. This leads to the state

$$
|\chi\rangle=c_{1}\left|\alpha_{1}\right\rangle \otimes\left|\beta_{1}\right\rangle+\sqrt{\sum_{k=2}\left|c_{k}\right|^{2}}|\alpha\rangle \otimes\left|\beta_{2}\right\rangle,
$$

where $|\alpha\rangle$ is a normalized vector. The measure assigned by this state to $\left|\beta_{2}\right\rangle\left\langle\beta_{2}\right|$ should be the sum of the original measures of the projectors that have coalesced into $\left|\beta_{2}\right\rangle\left\langle\beta_{2}\right|$.

Finally, because $\sum_{k=2}\left|c_{k}\right|^{2}=1-\left|c_{1}\right|^{2}$ we may write $\mu\left(\left|\beta_{1}\right\rangle\left\langle\beta_{1}\right|\right)=f\left(\left|c_{1}\right|^{2}\right)$. By parity of reasoning we may write down an analogous formula for the other projectors: $\mu\left(\left|\beta_{i}\right\rangle\left\langle\beta_{i}\right|\right)=f\left(\left|c_{i}\right|^{2}\right)$.

On the basis of our above observation about the relation between the measures induced by $|\psi\rangle$ and $|\chi\rangle$, respectively, we now find that

$$
f\left(\sum\left|c_{k}\right|^{2}\right)=\sum f\left(\left|c_{k}\right|^{2}\right)
$$

From this it follows that $f\left(\left|c_{k}\right|^{2}\right)=$ const. $\left|c_{k}\right|^{2}$, and in view of normalization

$$
\mu\left(P_{k}\right)=\left|c_{k}\right|^{2}
$$

This is the Born rule.

\section{Probability and modality}

As explained in the Introduction, modal interpretations understand $\mu$ as a probability: given the state $\psi$ in Hilbert space, exactly one of the projectors that are singled out as definite-valued by $\psi$ possesses the value 1 , and the chance that this value is taken by $P_{k}$ is given by $\left|c_{k}\right|^{2}$. In general there are more than one possibilities for the actual physical situation (defined by the values of the definite-valued observables), once the state in Hilbert space has been given; the state specifies a probability distribution over them. This probability quantifies our ignorance about the actually obtaining physical situation in cases in which we know the state in Hilbert space and have no additional information. It is also reflected in the relative frequencies with which physical properties occur in repetitions of situations corresponding to the same $\psi$. In other words, the probabilities occurring in the modal interpretation have the same status as classical probabilities and have the usual classical interpretations. That $\mu$ has this physical meaning in terms of probabilities and ignorance 
is clearly something that is not decided by the mathematical formalism itself (see for a dissenting voice (Zurek, 2005), and for a convincing critical analysis of this argument e.g. (Mohrhoff, 2004)). It is an interpretational postulate that should be judged on the basis of comparison with alternatives - we shall have more to say about this in the next section.

According to the modal interpretation the state in Hilbert space thus is about possibilities, about what may be the case; about modalities. But there is also a second aspect to $\psi$ : it is the theoretical quantity that occurs in the evolution equation, and its evolution governs deterministically how the set of definite valued quantities changes. This double role of $\psi$, on the one hand probabilistic and on the other dynamical and deterministic, is a well-known feature of the Bohm interpretation. As we have seen in section 2, the Bohm interpretation can be regarded as a specific version of the modal interpretation, namely one in which there is an a priori given definite-valued observable. As we see now, the double deterministic-and-probabilistic aspect of $\psi$ is typical of modal interpretations quite generally.

\section{One versus many worlds}

The no-collapse scheme by itself does not imply anything about probability: it just says that the Hilbert space state evolves unitarily. An interpretation, which is external to the formalism, must be supplied before anything can be stated about what the state represents. It is sometimes suggested, however, in opposition to this, that the no-collapse formalism is capable of providing its own interpretation (DeWitt and Graham, 1973, p. 168). What seems to be meant is the claim that there exists a simplest interpretation that does most justice to the symmetries inherent in the Hilbert space formalism. In particular, the suggestion is that, granted the usual interpretational links between eigenstates of observables and values of physical quantities, a superposition of such eigenstates should be interpreted as representing the joint existence of the corresponding values. This is the many-worlds idea: superpositions represent collections of worlds, in each one of which exactly one value - corresponding to one term from the superposition - of an observable is realized. The claim is that this many-worlds interpretation distinguishes itself by being simple and by possessing a natural fit to the formalism, respecting its symmetries.

In a superposition all terms occur in the same way, i.e. without any markers that single out one, or some, terms as corresponding to what actually is the case. The basic thought of the many-worlds interpretation is that this symmetry signifies that all terms correspond to reality in the same way: if one term refers to something actually existing, then so must all. The identification of any particular term as representing actuality is regarded as breaking the 
symmetry present in the state, and therefore as objectionable.

Let us have a closer look at this argument. It may be conceded that singling out any particular term from a superposition, and identifying it as the one referring to actuality, breaks the symmetry of the state. But do probabilistic interpretations really work this way; do they single out one term over the others? Consider the analogous situation in classical probability theory: the same train of thought applied there would also lead to the conclusion that all events to which a probability distribution assigns a value should be simultaneously realized, if we do not have an underlying deterministic theory. One would be led, also in the classical case, to a many worlds ontology as the one that best fits the probability formalism. But is this interpretation really simpler or more symmetric than the usual one? Answering this question requires comparing two different mappings (reference relations), with a mathematical event space as their common domain. The probabilistic mapping is from the event space to possibilities; whereas the many-worlds mapping maps all elements of the event space into realities. Apart from this difference in status of the elements of the ranges of the two mappings (possibility and reality), which as far as the mapping itself is concerned is just a difference in labels, everything is the same. It is therefore hard to see how there could be any difference in simplicity, naturalness or symmetry.

The impression that there nevertheless is such a difference evidently derives from the notion that the probabilistic interpretation identifies one of the possibilities as the actual one, and thus violates the symmetry that is present in the many-worlds option. But this notion is incorrect. Not singling out such a privileged event is precisely what makes an interpretation fundamentally probabilistic. The probabilistic option treats all elements of the probability space in exactly the same way, by mapping them to possibilities that may be realized - it does not tell us which possibility is realized. Each single element of the interpretation's range may correspond to reality. There is therefore the same symmetry as in the many-worlds option.

Still, there is a difference. In the probabilistic interpretation it is stipulated from the outset that exactly one possibility is realized. Even if there is symmetry with respect to which possibility this is, is it not true that this oneworld stipulation by itself introduces surplus structure that is not present in the many-worlds interpretation? I do not think this is right. There is perfect equivalence in the sense that the many-worlds interpretation is defined by the condition that each element of the measure space corresponds to an actual states of affairs, whereas the probabilistic alternative is defined by the condition that each element may correspond to the one actual (but unspecified) state of affairs. There is consequently no difference in the symmetry properties or simplicity of the interpretations, but rather a difference in the nature of their ranges: in the one case this is a collection of many real worlds, in the 
other it is a collection of candidates for the one real world. So, in the end the significant difference boils down to the difference between one and many - and it surely is not a principle of metaphysics or rational theory choice that many is simpler than one. General considerations concerning symmetry and simplicity do therefore not favor a many worlds interpretation over a probabilistic, modal, interpretation.

Let us briefly discuss a further general problem with the many-worlds idea, namely the well-known question of how to accommodate the notion of probability at all in a theory according to which it is certain that all possibilities will be actually realized. The dominant opinion among many-worlds adherents seems to have become that the quantum probabilities should be seen as subjective, in the sense of quantifying a subject's degree of belief about the future experiences of his splitting self (though not subjective in the sense of purely personal: The Born probabilities should come out as governing the objectively most rational choices). This Deutsch-Wallace line of argument (Deutsch, 1999; Wallace, 2003, 2005) proceeds from the assumption that a subject should be indifferent between terms in the total superposition that occur with equal 'weights', i.e. squared absolute values of the coefficients. This apparently presupposes a probabilistic conception of the quantum state - even though it is now probabilistic in the subjective sense. Indeed, it is a priori unclear why there should be unique rational expectations defined at all in a situation corresponding to a particular $\psi$ if we do not start out by assuming a probabilistic meaning of the wave function. And even if we do accept that there are measures of our credence hidden in the quantum formalism, it still is not self-evident that the symmetries of the quantum state are significant for them (the latter point is also made by Price (2006)). In our modal approach we did not face these problems, because we explicitly took an interpretational step and postulated a probabilistic meaning of $\psi$, and moreover required the probabilities to be definable in terms of $\psi$. It is this latter requirement that makes the symmetries in $\psi$ relevant for the probability assignment.

That the universe consists of many actual worlds, each one containing exactly one possible outcome of a process, actually does not play a role in the technical part of the Deutsch-Wallace argument. It is only the quantum state $\psi$ that enters into the reasoning, as said with the assumption that $\psi$ should govern rational expectations to start with. Any conclusions that can be drawn from such reasoning in the context of interpretations with many coexisting actual worlds, can surely also be drawn in the context of the more usual probabilistic construal (namely that only one possibility will be actually realized), or so it would seem. However, Wallace (2005) argues that the many worlds interpretation $i s$ essential here. In the course of defending the idea that a rational agent should be indifferent between outcomes that occur with equal weights in the superposition $\psi$ (he calls this principle equivalence), Wallace says: 
"I wish to argue that the Everett interpretation necessarily plays a central role in any such defence: in other interpretations, equivalence is not only unmotivated as a rationality principle but is actually absurd.

Why? Observe what equivalence actually claims: that if we know that two events have the same weight, then we must regard them as equally likely regardless of any other information we may have about them. Put another way, if we wish to determine which event to bet on and we are told that they have the same weight, we will be uninterested in any other information about them.

But in any interpretation which does not involve branching - that is, in any non-Everettian interpretation - there is a further piece of information that cannot but be relevant to our choice: namely, which event is actually going to happen? If in fact we know that $\mathrm{E}$ rather than $\mathrm{F}$ will actually occur, of course we will bet on E, regardless of the relative weights of the events".

It seems to me that this argument does not work. In reasoning about rational expectations - connected to subjective probabilities - about future events one standardly distinguishes 'admissible' from 'inadmissible' information. For example, David Lewis's 'Principal Principle' says that a rational agent should set his subjective probability equal to the objective chance of an event, unless there is (inadmissible) information about what is actually going to happen (Lewis, 1986). In other words, it is not a sound principle that if two events have the same objectively and rationally founded subjective probability, then we have to regard them as equally likely completely regardless of any other information we may receive. In our probability judgements we do not use information about the actual outcomes; if such information were to reach us we would of course adapt our expectations in spite of the probabilities. Conversely, no principle saying that our expectations should not change whatever further information reaches us should guide our search for the values of probabilities. If it did, no probability values other than 1 and 0 could ever be assigned, because in principle we might always receive information (e.g., by revelation) about whether things will or will not happen. This remains the case in the many-worlds scenario. It is true that all possibilities will become actual in this scenario, so that we cannot learn which event is actually going to happen. But we may still receive information about our actual future experiences, which according to Wallace are subject to uncertainty in the many-worlds universe because we ourselves split and do not know who of our successors we will become (this way of accommodating uncertainty and probability in the manyworlds interpretation is itself the subject of controversy - see (Greaves, 2004; Lewis, 2003, 2005; (Price, 2006)).

I conclude that the difference between one real world (and many possible ones) on the one hand, and many real worlds with subjective uncertainty injected into them on the other hand, is irrelevant for the justification of the form of Born rule. The Born formula can be derived from the requirement that the 
measure $\mu$ should be definable in terms of only $\psi$ (and should therefore not depend on anything else, like information about future events). The meaning of this $\mu$, be it in terms of modal probabilities or subjective many-worlds uncertainty, is something that cannot be derived but must be added in an interpretational step.

Let us now direct our attention to a more specific comparison of modal ideas and many worlds, relating to technical details.

One would perhaps expect the many worlds interpretation to follow the earlier explained modal way of fixing the definite observables in determining the worlds (each world corresponding to a different set of values of the definite observables), because a basic idea of the many-worlds interpretation is that the formalism is self-sufficient and that knowledge of the state is enough to obtain a full description of the universe. However, the dominant opinion among many-world adherents is different, namely that a privileged decomposition of the state is determined by the dynamical mechanism of decoherence. At first sight this decoherence recipe is almost identical to the one described in sections 2 and 3. Indeed, decoherence leads to a state of the general form

$$
|\psi\rangle=\sum_{k} c_{k}\left|\psi_{k}\right\rangle \otimes\left|E_{k}\right\rangle
$$

with $\left|\psi_{k}\right\rangle$ representing the part of the world undergoing decoherence, and $\left|E_{k}\right\rangle$ representing the decohering part (usually the environment of the system that is undergoing decoherence). This looks similar to Eq. 1. The difference is that the states $\left|E_{k}\right\rangle$ are not exactly orthogonal, so that Eq. 11 is not a biorthogonal decomposition. It is true that typically not only $\left\langle E_{i} \mid E_{j}\right\rangle \rightarrow 0$ when $t \rightarrow$ $\infty$, but that $\left\langle E_{i} \mid E_{j}\right\rangle \simeq 0$, if $i \neq j$, even very soon after the onset of the decoherence process: this process is very effective. Still, this inner product can never be assumed to really vanish at finite times. This means that the branches corresponding to the terms in Eq. 11 are not disjoint: there is interference between them. In the case of a biorthogonal decomposition the expectation value - taken in the total state - of any observable of the form $A \otimes I$ is the sum of contributions from the different branches; but this is not true in the case of the state of Eq. 11. In the latter case there are cross terms in addition to the contributions from the individual branches. Although these cross terms will typically be tremendously small, they are important from a conceptual point of view. They indicate that the total situation represented by the state cannot be viewed as a juxtaposition of independent alternatives or isolated worlds.

Another way of formulating the same point is that the Born probabilities of measurement results of observables of the form $A \otimes I$, calculated in the individual branches, when added with weights equal to the Born probabilities 
of those individual branches themselves, do not reproduce the probabilities of these outcomes in the total state. This is a violation of a consistency condition on the interpretation of $\left|c_{k}\right|^{2}$ as a probability both within the individual worlds and in the universe consisting of many worlds.

A second conceptual difficulty is that the way the state $|\psi\rangle$ has been decomposed in Eq.11 is not unique. A change of basis from $\left|\psi_{k}\right\rangle$ to a slightly different set of mutually orthogonal vectors will preserve the general form of Eq. 11, together with the almost orthogonality of the decohering states. So decoherence does not lead to a well-defined set of branches. Many-worlds proponents usually see this as an innocent form of vagueness: it is sufficient that on the macroscopic level the usual quantities, defined within observational precision, become definite - this is compatible with some leeway in the quantities that are definite on a microscopic scale. As Butterfield (2002) formulates it:

"...the ubiquity and astonishing efficiency of decoherence means that for all macrosystems ... the selected quantity will be very nearly unique - so that the vagueness will be unnoticeable by the standards of precision usual for macroscopic physics".

This may seem plausible, but it is not a solid result backed up by calculations. That is, at present there is no guarantee that the different possible choices of $\left|\psi_{k}\right\rangle$ that make it possible to write the state in the 'decoherent form' of (11), with $\left\langle E_{i} \mid E_{j}\right\rangle$ very small, are close to each other in Hilbert space. As Bacciagaluppi (2000) has shown in the context of modal interpretations, instabilities may occur in the biorthogonal decomposition, and sometimes this may lead to the selection of observables that are very different from the usual macroscopic observables one would expect to be selected. These results apply in particular to situations in which there are very many degrees of freedom, for example when macroscopic bodies are immersed in a decohering environment. It is true that these results have been rigourously derived only for the biorthogonal decomposition, but if $\left\langle E_{i} \mid E_{j}\right\rangle$ is very small instead of exactly zero, one should expect a similar behavior. This raises the general question of whether the decoherence scheme will be capable of always defining an adequate set of worlds, or definite outcomes of experiments. This is the same question that can be asked in the case of modal interpretations (cf. footnote 2).

In general, it seems that the technical difficulties which have been suggested to exist for modal interpretations based on the biorthogonal decomposition (in conditions with continuous or very many degrees of freedom (Bacciagaluppi, 2000; Donald, 1998)), should also be taken seriously in interpretations based on the idea that decoherence singles out a preferred decomposition of the state. These difficulties have been investigated in some detail only within the modal framework, because here there are precise mathematical rules that define the 
definite valued observables. However, although there is more 'slippage' in the decoherence scheme, the problem of instability requires a solution in this context as well. One should be careful here to distinguish between two different questions. In standard treatments of decoherence one starts by writing the state as a superposition of eigenstates of an a priori given preferred observable, often position; and then shows that these eigenstates become correlated, through the interaction with the surroundings, with almost orthogonal environment states. The question we are facing here, however, is whether the total state, after having undergone decoherence, defines adequate physical quantities. The uncontroversial reply to the first question does not answer the second; and it is to this second question that the above questions pertain.

A final point is that the use of approximations, which is very common in decoherence approaches, runs the risk of getting into vicious circles. In particular, the motivation for neglecting 'small' components of $\psi$ seems to presuppose a probabilistic interpretation of the coefficients with which these components occur. But this would vitiate a deduction of the Born rule. Indeed, the Born rule or something equivalent to it would in this case already be used in the definition of the definite events to which the Born probabilities are later to be assigned.

In summary, both from the viewpoint of general considerations and from that of more detailed quantum mechanical arguments there seems little reason to prefer many-worlds interpretations over modal ones. Decoherence is not an obvious help for the many-worlds scheme - if the problems just mentioned can be solved, it still remains unclear why decoherence would help the many-worlds theorist and would not be available to adherents of modal ideas.

\section{Conclusion}

The Hilbert space formalism of quantum mechanics restricts possible interpretations in the following sense: if we stipulate that definite-valued quantities and a probability measure over them should be definable from the quantum state and the Hilbert space tensor product structure for the system and its environment, this leads to unique expressions both for these definite quantities and the measure. That the state vector should be thus interpreted in terms of definite quantities and probabilities is not something that can be derived from the mathematical formalism - it is an interpretational choice. Modal interpretations implement this choice by postulating that the quantum state represents possibilities of which only one is realized in physical reality.

What the definite quantities turn out to be obviously depends on what we stipulate about the elements in the formalism on which these quantities are 
to depend. Here we have focused on what follows from the requirement of definability from only the state and the bipartite tensor product structure of Hilbert space. Other stipulations are possible while staying within the general framework of the modal programme, which is characterized by objectivity (description of the world through objective physical quantities) and a fundamental role for probability. These alternative options deserve further investigation.

\section{References}

Bacciagaluppi, G. (2000). Delocalized properties in the modal interpretation of a continuous model of decoherence. Foundations of Physics, 30, 1431-1444.

Bacciagaluppi, G. and Hemmo, M. (1996). Modal interpretations, decoherence, and measurements. Studies in the History and Philosophy of Modern Physics, 27, 239-277.

Barnum, H. (2003). No-signalling-based version of Zurek's derivation of quantum probabilities; arXiv:quant-ph/0312150.

Caves, C.M. (2005). Notes on Zurek's derivation of the quantum probability rule. Available at info.phys.unm.edu/ caves/reports/ZurekBornderivation.pdf

Bene, G. and Dieks, D. (2002). A perspectival version of the modal interpretation of quantum mechanics and the origin of macroscopic behavior. Foundations of Physics, 32, 645-671; arXiv:quant-ph/0112134.

Bub, J. and Clifton, R. (1996). A uniqueness theorem for 'no collapse' interpretations of quantum mechanics. Studies in the History and Philosophy of Modern Physics, 27, 181-219.

Bub, J. (1997). Interpreting the Quantum World. Cambridge: Cambridge University Press.

Bub, J., Clifton, R. and Goldstein, S. (2000). Revised proof of the uniqueness theorem for 'no collapse' interpretations of quantum mechanics. Studies in the History and Philosophy of Modern Physics, 31, 95-98.

Butterfield, J.N. (2002). Some worlds of quantum theory. In R. Russell et al. (Eds.), Quantum Mechanics: Scientific perspectives on divine action 5, 111140. Notre Dame: University of Notre Dame Press; arXiv:quant-ph/0105052.

Deutsch, D. (1999). Quantum theory of probability and decisions. Proceedings of the Royal Society of London, A455, 3129-3137; arXiv:quant-ph/9906015.

DeWitt, B.S. and Graham, N. (1973). The Many-Worlds Interpretation of Quantum Mechanics. Princeton: Princeton University Press.

Dieks, D. (1989a). Quantum mechanics without the projection postulate and its realistic interpretation. Foundations of Physics, 19, 1395-1423.

Dieks, D. (1989b). Resolution of the measurement problem through decoherence of the quantum state. Physics Letters, A142, 439-446.

Dieks, D. (1994). The modal interpretation of quantum mechanics, measurement and macroscopic behaviour. Physical Review, D49, 2290-2300. 
Dieks, D. (1995). Physical motivation of the modal interpretation of quantum mechanics. Physics Letters, A197, 367-371.

Dieks, D. (2005). Quantum mechanics: an intelligible description of objective reality? Foundations of Physics, 35, 399-416.

Dieks, D. and Vermaas, P. (Eds.) (1998). The Modal Interpretation of Quantum Mechanics. Dordrecht: Kluwer Academic Publishers.

Donald, M.J. (1998). Discontinuity and continuity of definite properties in the modal interpretation. In Dieks and Vermaas (1998, 213-222).

Greaves, H. (2004). Understanding Deutsch's probability in a deterministic universe. Studies in the History and Philosophy of Modern Physics, 35, 423456; arXiv:quant-ph/0312136.

Kochen, S. (1985). A new interpretation of quantum mechanics. In P. Lahti and P. Mittelstaedt (Eds.), Symposium on the Foundations of Modern Physics, 151-169. Singapore: World Scientific.

Lewis, D. (1986). A subjectivist's guide to objective chance. In Philosophical Papers, Volume II, 83-132. Oxford: Oxford University Press.

Lewis, P. (2003). Deutsch on quantum decision theory. Available at http://philsci-archive.pitt.edu/archive/00001350/.

Lewis, P. (2005). Probability in Everettian quantum mechanics. Available at http://philsci-archive.pitt.edu/archive/00002716/.

Mohrhoff, U. (2004). Probabilities from envariance? International Journal of Quantum Information, 2, 221-230; arXiv:quant-ph/0401180.

Price, H. (2006). Probability in the Everett world: comments on Wallace and Greaves. Available at http://philsci-archive.pitt.edu/archive/00002719/.

Schlosshauer, M. (2004). Decoherence, the measurement problem, and interpretations of quantum mechanics. Reviews of Modern Physics, 76, 12671305; arXiv:quant-ph/0312059.

Schlosshauer, M. and Fine, A. (2005). On Zurek's derivation of the Born rule. Foundations of Physics, 35, 197-213; arXiv:quant-ph/0312058.

van Fraassen, B. (1981). A modal interpretation of quantum mechanics. In E. Beltrametti and B. van Fraassen (Eds.), Current Issues in Quantum Logic, 229-258. New York: Plenum.

van Fraassen, B. (1991). Quantum Mechanics. Oxford: Clarendon Press.

Vermaas, P.E., and Dieks, D. (1995). The modal interpretation of quantum mechanics and its generalization to density operators. Foundations of Physics, 25, 145-158.

Wallace, D. (2003). Everettian rationality: defending Deutsch's approach to probability in the Everett interpretation. Studies in History and Philosophy of Modern Physics, 34, 415-439; arXiv:quant-ph/0303050.

Wallace, D. (2005). Quantum probability from subjective likelihood: improving on Deutsch's proof of the probability rule. Available at http://philsci-archive.pitt.edu/archive/00002302/,

Zurek, W. H. (1993). Preferred states, predictability, classicality and the environment-induced decoherence. Progress of Theoretical Physics, 89, 281312 . 
Zurek, W. H. (2005). Probabilities from entanglement, Born's $p_{k}=\left|\psi_{k}\right|^{2}$ rule from envariance. Physical Review, A71, 052105; arXiv:quant-ph/0405161 